\title{
Alterstice
}

Revue internationale de la recherche interculturelle

International Journal of Intercultural Research

Revista International de la Investigacion Intercultural

\section{Socialisation pour l'acculturation : la dynamique de la transmission familiale de l'adaptation en pays d'accueil}

\section{Colette Sabatier}

Volume 3, numéro 1, 2013

Les diversités au coeur de la recherche interculturelle : vers de nouvelles perspectives

URI : https://id.erudit.org/iderudit/1077498ar

DOI : https://doi.org/10.7202/1077498ar

Aller au sommaire du numéro

Éditeur(s)

Alterstice

ISSN

1923-919X (numérique)

Découvrir la revue

Citer cet article

Sabatier, C. (2013). Socialisation pour l'acculturation : la dynamique de la transmission familiale de l'adaptation en pays d'accueil. Alterstice, 3(1), 47-60. https://doi.org/10.7202/1077498ar

\section{Résumé de l'article}

La socialisation des adolescents issus de l'immigration soulève pour leurs parents des défis particuliers. Comment transmettre sa culture et inculquer à l'adolescent l'attachement à la famille et ses valeurs et ainsi s'assurer la survie du groupe familial, et en même temps accompagner les enfants dans un processus d'adaptation au pays d'accueil et respecter leur désir d'individuation et d'autonomie ? Une socialisation réussie suppose quatre conditions : un contexte, un sujet apprenant malléable et désireux d'apprendre, des agents de socialisation et en phase avec l'environnement et enfin une communauté apprenante. Cet article présente cinq recherches qui étudient les processus conjoints de transmission parentale des valeurs de la société d'accueil et de la culture d'origine et examinent les articulations entre les différentes sources d'influence, aux niveaux sociétal et familial, dans le processus de transmission des valeurs culturelles, celles de la société dans sa globalité et celles de la culture minoritaire. 


\title{
7
}

alterstice

ARTICLE THÉMATIQUE

Socialisation pour l'acculturation : la dynamique de la transmission familiale de l'adaptation en pays d'accueil

\author{
Colette Sabatier ${ }^{1}$
}

\section{Résumé}

La socialisation des adolescents issus de l'immigration soulève pour leurs parents des défis particuliers. Comment transmettre sa culture et inculquer à l'adolescent l'attachement à la famille et ses valeurs et ainsi s'assurer la survie du groupe familial, et en même temps accompagner les enfants dans un processus d'adaptation au pays d'accueil et respecter leur désir d'individuation et d'autonomie? Une socialisation réussie suppose quatre conditions : un contexte, un sujet apprenant malléable et désireux d'apprendre, des agents de socialisation et en phase avec l'environnement et enfin une communauté apprenante. Cet article présente cinq recherches qui étudient les processus conjoints de transmission parentale des valeurs de la société d'accueil et de la culture d'origine et examinent les articulations entre les différentes sources d'influence, aux niveaux sociétal et familial, dans le processus de transmission des valeurs culturelles, celles de la société dans sa globalité et celles de la culture minoritaire.

\section{Rattachement de l'auteure}

${ }^{1}$ Laboratoire Psychologie, Santé et Qualité de Vie, Université Bordeaux Segalen, Bordeaux, France

\section{Correspondance}

colette.sabatier@u-bordeaux2.fr

\section{Mots clés}

acculturation; relation parents-enfants; transmission; adolescents; immigrants

\section{Pour citer cet article :}

Sabatier, C. (2013). Socialisation pour l'acculturation : la dynamique de la transmission familiale de l'adaptation en pays d'accueil. Alterstice, 3(1), 47-60. 


\section{Introduction}

Comme le souligne, si justement, Vatz-Laaroussi (2001), toute la famille est concernée par l'acculturation. Les parents ont immigré pour améliorer leur qualité de vie ainsi que celle des membres de leur famille qui les ont accompagnés dans leur mouvement migratoire ou de la famille qu'ils ont créée une fois installés sur place. Ils ont vécu les processus d'acculturation et ont fait des efforts pour s'adapter. De ce fait, ils peuvent vouloir à la fois transmettre leur bagage culturel, encourager l'adaptation à la société d'accueil et transmettre leurs savoir-faire reliés à l'adaptation (Sabatier, 1991). Les parents investissent dans leurs enfants et tentent de gérer leur intégration sociale aussi bien dans leur groupe culturel que dans la société d'accueil en général (Sabatier et Berry, 2008). Par leurs efforts d'adaptation et leurs comportements culturels, les parents sont pour leurs enfants des modèles de compétence et d'adaptabilité. Les enfants et les adolescents observent leurs parents faire des efforts pour développer de nouvelles compétences professionnelles, apprendre une langue et découvrir des moyens d'adaptation à la société d'accueil, avec ses possibilités et ses écueils. De cette expérience, ils peuvent en retenir autant les éléments positifs que les éléments plus éprouvants. Mes travaux sur l'adaptation des adolescents issus de l'immigration explorent plusieurs facettes de cette question de la dynamique familiale de l'acculturation en interrogeant les processus de transmission familiale.

L'étude de la dynamique familiale de l'acculturation et de la transmission est un thème au carrefour de trois approches : 1) la psychologie développementale, notamment la psychologie sociale développementale et l'approche écologique du développement humain, qui situe le développement au sein d'interactions complexes entre différents paliers de l'environnement social (Sabatier, 1999); 2) les relations familiales, notamment les recherches sur la transmission familiale et culturelle et 3) la psychologie interculturelle de l'acculturation qui propose plusieurs modèles, celui que nous adoptons étant le modèle AIMS (Assimilation, Intégration, Marginalisation et Séparation) de Berry avec qui nous avons travaillé (Sabatier et Berry, 1994).

Une transmission sociale réussie, selon Troadec (2011), suppose quatre conditions : un contexte, un sujet apprenant suffisamment malléable et désireux d'apprendre, des agents de socialisation qui sont en principe en phase avec l'environnement social plus général et enfin une communauté apprenante, autrement dit une collectivité de pairs qui reçoivent les mêmes messages et qui sont également désireux d'apprendre. Les processus de transmission impliquent des courroies de transmission (Schönpflug, 2009): stratégies et volontés de transmettre, perceptions par l'apprenant des valeurs et identités auxquelles on voudrait qu'il adhère et qu'on voudrait qu'il s'approprie et enfin acceptation par l'apprenant de ces valeurs et identités.

Du point de vue du sujet apprenant, l'essentiel de mes recherches porte sur des adolescents, autrement dit sur une période de la vie où la tâche de développement est l'individualisation plutôt que la reproduction des valeurs. Les adolescents sont a priori peu enclins à accepter les valeurs de leurs aînés et cherchent à élaborer ou à tester les leurs, ce qui rend l'étude de la transmission des valeurs à cette période de la vie d'autant plus intéressante et pertinente. Du point de vue du contexte, mes recherches sur la socialisation des adolescents des minorités culturelles est un cas intéressant, où la conjonction entre les valeurs et pratiques de la société en général (le macrosystème) et l'environnement des familles (les microsystèmes) ne va pas de soi, sans pour autant que ces valeurs et pratiques soient en opposition ou en conflit. L'étude de la transmission dans le cas de disjonction potentielle entre les différentes instances de socialisation est plutôt rare, mais intéressante pour en comprendre les processus et son efficacité (Kelley et De Graaf, 1997; Knafo, 2003). Pour notre part, nous avons stipulé que plusieurs sources d'influence, celles de la société d'accueil, des groupes sociaux environnants et des parents, concourent à la socialisation pour l'acculturation selon des processus interactifs complexes.

Après avoir exposé, dans les deux premières parties de cet article, les fondements théoriques de mes recherches, je présente les résultats qui se dégagent de cinq d'entre elles. Les deux premières portent sur les processus conjoints de transmission parentale des valeurs de la société d'accueil et de la culture d'origine. Les trois suivantes examinent les articulations entre les différentes sources d'influence, aux niveaux sociétal et familial, dans le processus de transmission des valeurs culturelles, celles de la société dans sa globalité et celles de la culture minoritaire.

Alterstice - Revue Internationale de la Recherche Interculturelle, vol. 3, $n^{\circ} 1$ 


\section{La socialisation culturelle entre transmission et évolution : processus et conditions}

L'objectif général de la socialisation parentale est de permettre aux groupes sociaux d'optimiser leurs ressources et leurs savoir-faire. La transmission culturelle est essentielle pour l'organisation des sociétés et des groupes, mais en même temps elle doit permettre l'adaptation à de nouvelles contraintes de l'environnement et la flexibilité. Sans cette transmission, il n'y aurait pas de continuité dans les savoirs d'une génération à l'autre. Chaque génération devrait alors rebâtir ses connaissances et reconstruire un socle de valeurs permettant le contrat social qui lie les individus et leur permet de vivre ensemble et de partager une destinée commune. II en résulterait un coût énorme pour les sociétés. En contrepartie, aucun groupe n'a intérêt à ce que ses savoirs ou ses valeurs se perpétuent à I'identique. La socialisation se situe donc entre deux pôles, celui de la conservation à travers les générations des compétences effectives et celui de l'acquisition de nouvelles compétences pour optimiser la qualité de la vie et l'adaptation à un environnement sans cesse changeant.

La continuité des valeurs, cruciale pour le fonctionnement à long terme d'une société, est liée à l'ancrage culturel, à la mémoire collective et aux mémoires individuelles au sein des familles et du groupe. Cette continuité repose sur l'enculturation, c'est-à-dire les pratiques destinées à faire entrer la génération suivante dans le système culturel. Les changements de valeurs et d'attitudes sont fonctionnels, ils favorisent l'innovation, la créativité et l'adaptation à de nouvelles circonstances. Le changement de valeurs provient de deux sources: d'une part, les changements internes, économiques, sociaux ou culturels d'une société (changement social) et, d'autre part, le contact avec d'autres modes culturels de vie (acculturation). Ces changements internes et ces contacts ont existé de tout temps, mais ils s'accentuent avec les nouveaux moyens de communication et de transport.

Comment s'opère la transmission entre continuité et évolution, particulièrement dans le contexte d'une double transition, celle de la famille immigrée qui doit s'intégrer dans la société d'accueil et celle du sujet apprenant, l'adolescent né dans le pays où ses parents ont immigré? Quelles en sont les courroies de transmission, compte tenu du jeu des inter-influences entre les différentes sources de socialisation?

Du côté des sujets apprenants, ici les adolescents, les recherches montrent que leur perception et leur reconnaissance personnelle des valeurs de leurs propres parents sont autant, voire plus, déterminantes dans leur adoption des valeurs et des comportements que les valeurs, attitudes et comportements des parents eux-mêmes (Alessandri et Wozniak, 1987; Okagaki et Bevis, 1999). Du côté des parents, les recherches montrent que les parents font la distinction entre leurs propres attitudes et celles qu'ils souhaitent que leurs enfants adoptent (Youniss, 1994). Autrement dit, les parents sont capables de faire la distinction entre ce qu'ils souhaitent pour euxmêmes, parce qu'ils se sont construits ainsi, ce qu'ils projettent pour leurs enfants, et ce qu'ils souhaitent leur inculquer à la fois comme capacité d'adaptation à de nouvelles situations - dont la modernité - et comme conservation de la culture (Sabatier, 1991; Sabatier et Holveck, 2001). Ainsi, pour élucider les processus de transmission des attitudes des parents à leurs enfants, il faut tenir compte de la disjonction possible entre les attitudes des parents pour eux-mêmes et leurs objectifs pour leurs enfants, ainsi que des mécanismes de perception des adolescents de ces attitudes et objectifs.

Pour qu'une transmission soit efficace, il faut que les enfants reconnaissent et identifient correctement les valeurs et identités de leurs parents, mais il faut aussi que ces valeurs et attitudes représentent pour eux des offres recevables et attrayantes et qu'elles aient du sens pour eux (Schönpflug et Bilz, 2009). Les individus ont tendance à davantage endosser les valeurs des personnes auxquelles ils sont attachés et qui représentent des modèles positifs que celles des personnes avec qui les relations sont tendues ou qui ne bénéficient d'aucun prestige social. Les parents offrent un point d'ancrage, canalisent le développement et servent de base solide pour l'exploration de l'identité. La qualité de la relation adolescent-parent et l'attachement de l'enfant à ses parents agissent comme des courroies de transmission qui favorisent le relais entre les valeurs des parents et celles de l'adolescent. 


\section{Dynamiques de l'acculturation et de l'identité, entre continuité et rupture}

Développer des liens avec la société d'accueil, acquérir les compétences sociales nécessaires à une participation pleine dans la société d'installation et en même temps garder contact avec sa famille et son réseau social est une tâche développementale cruciale pour les adolescents issus de l'immigration. Depuis l'œuvre fondatrice de Child en 1943 sur l'identité duelle impossible des Italiens nés aux États-Unis, de nombreux travaux ont examiné la distance culturelle entre les parents et les immigrants. Plusieurs y ont vu une source de malaise et de difficultés pour les adolescents (Birman, 2006; Kwak et Berry, 2001; Lau et collab., 2005; Phinney et Ong, 2002; Sam et Virta, 2002). Cependant, toutes les recherches qui ont sérieusement analysé cette hypothèse n'ont montré aucun effet délétère de cette distance. La distance culturelle semble bien être une donnée de l'évolution entre les générations qui ne pose pas en soi de difficulté. Des recherches plus récentes se sont concentrées sur trois points plus prometteurs, les processus de socialisation effectués par les parents pour l'intégration dans la société d'accueil (Phinney et Chavira, 1995; Sabatier, 2008), la transmission par les parents de leurs attitudes d'acculturation et de leur identité (Nauck, 2001) et enfin le rôle respectif de chaque parent dans les processus d'acculturation (Costigan et Dokis, 2006; Killian et Hegtvedt, 2003; Sabatier et Berry, 2008).

À partir des théories de l'acculturation de Berry (Sabatier et Berry, 1994) et des théories de l'identité culturelle des minorités ethniques de LaFromboise, Coleman, et Gerton (1993) et de Phinney, Horenczyk, Liebkind et Vedder (2001), j'ai combiné les deux orientations de l'identité culturelle, à savoir l'identité ethnique et l'identité française, en stipulant que ces deux identités peuvent avoir différentes combinaisons selon les choix et les représentations des individus, mais aussi ceux des groupes minoritaires ou ceux imposés par les groupes majoritaires ou les sociétés d'accueil. Ces combinaisons sont plus complexes et subtiles que le dilemme de la continuité et de la rupture. Dans le cadre de la théorie de Marcia (1966) et de Phinney (1990) de la construction identitaire à l'adolescence et tout au long de la vie adulte, spécialement du jeune adulte, deux composantes de l'identité ont été considérées: l'affirmation identitaire (je suis conscient d'être, ou fier d'être, etc.) et l'exploration, c'est-à-dire le questionnement autour de l'identité et l'interrogation sur ses implications positives ou négatives.

\section{Études sur la transmission parentale et les rôles spécifiques du père et de la mère}

\section{Au niveau de la transmission de l'identité et des attitudes d'acculturation}

Dans une première recherche, deux hypothèses simultanées ont été explorées, celle bien étayée du fossé entre générations et celle moins documentée de la transmission des attitudes et des identités. Nous avons examiné ces hypothèses avec chacun des deux parents, ce qui a permis de repérer les rôles respectifs des pères et des mères dans ce processus de transmission. Nous avons interrogé 447 triades adolescent-mère-père sur leurs attitudes d'acculturation et leur identité. Les adolescents, âgés en moyenne de 15 ans et demi (de 11 à 19 ans), sont nés dans le pays d'accueil, alors que leurs parents ont immigré à l'âge adulte, soit 248 triades vers la France et 199 vers le Canada. Chaque membre a répondu à plusieurs questionnaires qualitatifs et quantitatifs au cours d'entrevues individuelles menées par des intervieweurs de la même origine ethnique qu'eux.

Nous avons adopté pour les mesures de l'identité culturelle et pour l'acculturation le principe du format bi-axial, c'est-à-dire une question ou une série de questions concernant l'orientation vers la société d'accueil, l'autre, l'orientation vers la culture d'origine, le tout sur une échelle de 1 à 5 . L'identité culturelle (nationale et ethnique) a été mesurée par deux questions (une par orientation culturelle), les attitudes d'acculturation envers la société d'accueil par huit questions et les attitudes envers leur groupe ethnique par quinze (voir Berry et Sabatier, 2010; Sabatier et Berry, 2008).

Une série de tests $t$ pour groupes appariés a permis d'étudier à la fois les différences systématiques entre les adolescents et chacun des deux parents, et les corrélations entre eux, compte tenu du fait que les adolescents et leurs parents font partie du même environnement familial. 
Tableau 1. Différences et corrélations entre les attitudes et les identités des adolescents et chacun de leurs deux parents

\begin{tabular}{lcccccccc}
\hline & \multicolumn{3}{c}{$\begin{array}{c}\text { Attitudes d'acculturation } \\
\text { ethnique }\end{array}$} & \multicolumn{2}{c}{ nationale } & \multicolumn{2}{c}{ ethnique } & \multicolumn{2}{c}{ nationale } \\
\hline Moyennes & $m$ & ét & $m$ & ét & $m$ & et & $m$ & ét \\
adolescents & 4,09 & 0,62 & 3,43 & 0,72 & 3,94 & 1,00 & 3,30 & 1,06 \\
mères & 4,42 & 0,50 & 3,18 & 0,71 & 4,73 & 0,69 & 2,36 & 1,57 \\
pères & 4,46 & 0,47 & 3,27 & 0,77 & 4,80 & 0,57 & 2,55 & 1,67 \\
T-test & $t$ & sign. & $t$ & sign. & $t$ & sign. & $t$ & sign. \\
et mères & $-12,29$ & $* * *$ & 5,91 & $* * *$ & $-14,64$ & $* * *$ & 11,46 & $* * *$ \\
et pères & $-12,44$ & $* * *$ & 3,66 & $* * *$ & $-16,44$ & $* * *$ & 9,06 & $* * *$ \\
Corrélations & $r$ & sign. & $r$ & sign. & $r$ & sign. & $r$ & sign. \\
et mères & 0,49 & $* * *$ & 0,23 & $* * *$ & 0,13 & $* *$ & 0,17 & $* * *$ \\
et pères & 0,36 & $* * *$ & 0,27 & $* * *$ & 0,09 & $*$ & 0,24 & $* * *$ \\
\hline
\end{tabular}

$* p<0,05 ; * * p<0,01 ; * * *<<0,001$

Les résultats aux quatre mesures (tableau 1) corroborent la coexistence des différences de génération et des processus de transmission. Les adolescents sont en général plus orientés que leurs deux parents vers la société d'accueil et un peu moins vers leur culture. Ces différences, tant pour leurs attitudes et que pour l'identité, sont en lien avec celles de chacun de leurs deux parents (corrélations significatives). Une analyse (régression multiple; cf tableau 2) évaluant les rôles respectifs des deux parents après avoir contrôlé le sexe de l'enfant et le pays d'accueil montre que si les deux parents contribuent à cette transmission, l'influence relative de la mère est plus importante en ce qui concerne l'orientation vers la culture d'origine (attitudes et identité) et celle du père plus importante en ce qui concerne l'acculturation et l'identité en lien avec la société d'accueil. En outre, les résultats indiquent une transmission relativement claire dans le cas des attitudes d'acculturation vis à vis de la culture d'origine avec une transmission plus modeste mais significative en ce qui concerne les attitudes envers la société d'accueil et pour les deux dimensions de l'identité culturelle. Des analyses statistiques plus rigoureuses permettant de contrôler le jeu des interinfluences, mais plus complexes, à savoir l'analyse de dominance (Cosnefroy et Sabatier, 2011), confirment ces résultats. Le sexe de l'adolescent ne semble pas avoir d'influence particulière, et le père et la mère n'ont pas d'influence différenciée entre fils et filles.

Tableau 2. Influences respectives de chacun des deux parents (régression hiérarchique)

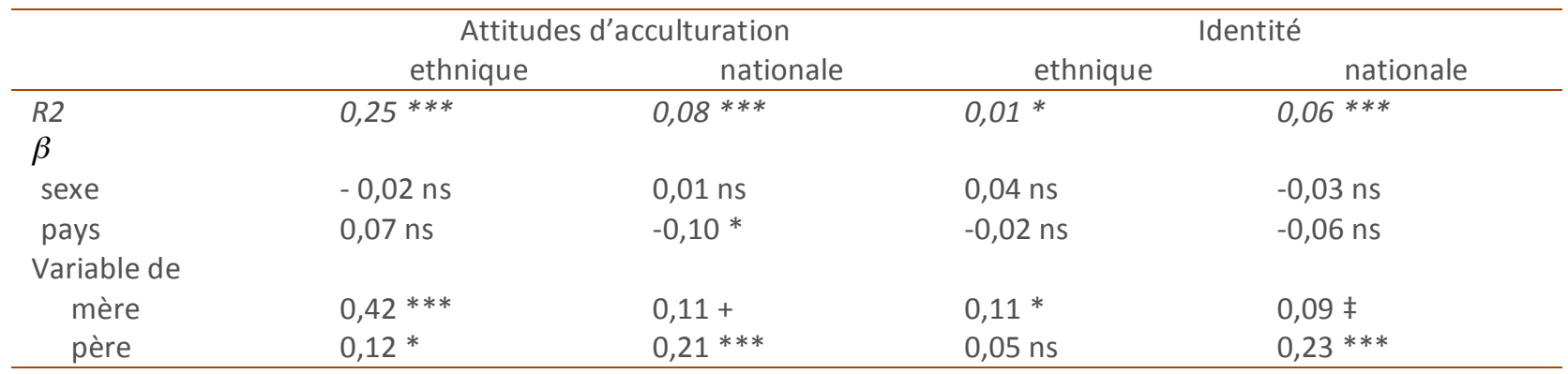

$\ddagger p<0,10 ; * p<0,05 ; * * p<0,01 ; * * * p<0,001$

En somme, les parents transmettent leurs attitudes et leurs identités, mais il semble plus facile de transmettre une attitude marquante, qui agit comme un ciment identitaire dans un groupe minoritaire qui a besoin de rehausser son image, qu'une attitude plus neutre, envers un groupe dont la taille est plus importante, et qui de ce fait englobe une large palette de valeurs et de références sociales (ce qui est le cas des sociétés d'accueil) sans pour autant que son unité soit menacée. 


\section{Au niveau de la perception de la discrimination}

En nous situant dans le cadre de la psychologie développementale, nous pensons que la perception de la discrimination évolue avec l'âge. C'est faire acte de maturité que d'être capable de repérer la discrimination là où il y en a. Cette attitude permet de lutter contre ces actes, et également de s'en protéger en les considérant comme un fait de société et non comme une diminution de soi à laquelle l'individu doit adhérer. La perception de la discrimination a de multiples facettes et enjeux. Elle se joue sur de nombreux registres. Selon le modèle développemental de Brown et Bigler (2005), trois conditions sont nécessaires : des compétences socio-cognitives qui permettent de distinguer ce qui est juste de ce qu'il ne l'est pas et d'élaborer la notion d'égalité de traitement et d'équité; un contexte social selon les forces et les groupes en présence, le climat des relations intergroupes dans la société et enfin les caractéristiques personnelles des individus, la force de leur identité, leurs valeurs pour l'équité ou la compétition, leur histoires affectives personnelles. Trois modèles explicatifs de transmission familiale de la perception de la discrimination sont présents dans la littérature sans qu'ils aient été confrontés les uns aux autres. Le premier porte sur la transmission directe: les parents qui subissent de la discrimination peuvent transmettre de façon incidente ou délibérée leur vision de la société. C'est le modèle de la reproduction de la fatalité. Le plus souvent ce sont les pères, qui sont davantage en contact avec la société d'accueil, qui transmettent cette vision à leurs fils (Nauck, 2001). Le deuxième modèle est celui proposé par De Vos (1980) et Lewin (1936), c'est celui d'un enseignement délibéré d'analyse de la discrimination afin de permettre aux enfants et aux adolescents de construire un sens de soi distinct de l'image négative de leur groupe véhiculée dans la société environnante. C'est le modèle de la socialisation ethnique/raciale. Ce modèle est adopté par exemple par les parents militants noirs américains pour transmettre à leurs enfants une fierté raciale. Le troisième modèle est le modèle de la protection affective. En soutenant affectivement leurs enfants, les parents les aident à se construire une image de soi positive et à se sentir bien dans leur peau, ce qui les oriente vers une vision plus optimiste de la vie et les éloignent d'une perception négative du monde (Jasinskaja-Lahti et Liebkind, 2001).

Dans une autre recherche (Sabatier et Berry, 2009), qui reprend la même base de données que la recherche précédente, nous avons analysé le rôle du père et de la mère sur la perception de la discrimination personnelle ou de groupe des adolescents. Nous avons examiné trois niveaux d'influence parentale selon les trois modèles explicatifs. En premier lieu, les attitudes d'acculturation de chacun des deux parents (modèle de la transmission de la fatalité), en deuxième lieu leurs pratiques délibérées de l'enseignement du racisme et de la discrimination (modèle de la socialisation ethnique/raciale) et en troisième lieu le climat relationnel parents-adolescents (modèle de la protection affective).

Tableau 3. Influences respectives de chaque parent sur la perception de la discrimination

\begin{tabular}{|c|c|c|}
\hline & personnelle & de groupe \\
\hline$R 2$ & 0,30 & 0,26 \\
\hline Sexe & $0,24 * * *$ & $0,19 * *$ \\
\hline Âge de l'adolescent & $-0,16 * *$ & \\
\hline \multicolumn{3}{|l|}{ Orientation culturelle des parents } \\
\hline \multirow{3}{*}{$\begin{array}{ll}\text { père: } & \text { ethnique } \\
& \text { nationale } \\
\text { mère: } & \text { ethnique }\end{array}$} & $0,16 *$ & \\
\hline & & $-0,15 * *$ \\
\hline & & $0,19 * *$ \\
\hline \multicolumn{3}{|l|}{ Enculturation des parents } \\
\hline père conversation relation intergroupe & $0,25 * * *$ & $0,16 *$ \\
\hline \multicolumn{3}{|l|}{ Climat relationnel avec les parents } \\
\hline père harmonie culturelle & $-0,20 * *$ & $-0,27 * * *$ \\
\hline \multirow{2}{*}{$\begin{array}{l}\text { soutien/aide école } \\
\text { autoritaire }\end{array}$} & $-0,16 * *$ & \\
\hline & $0,13 *$ & \\
\hline
\end{tabular}


Les résultats de la régression multiple qui apparaissent dans le tableau 3 montrent que les trois modèles explicatifs sont valables. On retrouve un poids explicatif pour chacun d'entre eux. Il existe du côté de la famille des éléments qui sensibilisent les adolescents à la perception de la discrimination personnelle et de la discrimination de groupe et des éléments qui atténuent cette perception. Ces résultats montrent en outre que les pères et les mères exercent des rôles complémentaires, avec l'impression que les pères de ce point de vue auraient plus de poids. Ces processus d'influence sont relativement identiques pour les garçons et les filles, bien que les garçons perçoivent plus de discrimination, et que les adolescents plus âgés moins de discrimination personnelle.

\section{Études sur les courroies de transmission}

\section{Étude sur l'influence de la perception des adolescents et des objectifs d'acculturation des mères}

Avec un groupe de 74 élèves de sixième, marocains de seconde génération, et leurs mères, nous avons examiné les processus de transmission des attitudes d'acculturation aux adolescents, en analysant comment les perceptions de l'enfant interviennent au sein de ce processus (Boutry-Avezou et Sabatier, 2006; Sabatier et Avezou-Boutry, 2012). Les adolescents et leurs mères ont été interrogés sur leurs attitudes d'acculturation selon le modèle AIMS de Berry (2006) en adoptant la procédure des quatre échelles distinctes, une pour chaque option d'acculturation. Dans cette recherche, trois attitudes sont considérées: l'intégration (orientation positive vers la société française tout en gardant une attitude positive envers sa propre culture), l'assimilation (orientation préférentiellement vers la société française aux dépens de l'attachement à sa propre culture) et la séparation (orientation préférentiellement vers la culture d'origine). Nous avons distingué, chez les mères, leurs attitudes personnelles en tant qu'adulte et leurs objectifs d'acculturation pour leur enfant, autrement dit, leurs aspirations pour leur enfant en termes d'attitudes d'acculturation. Du côté des adolescents, nous avons distingué leurs attitudes d'acculturation et leurs perceptions des attitudes et des objectifs d'acculturation de leur mère.

Rejoignant en cela certains travaux portant sur la transmission des valeurs (Alessandri et Wozniak, 1987; Okagaki et Bevis, 1999), nous pensons que les adolescents perçoivent adéquatement les attitudes et les objectifs des mères et que la perception de ces attitudes et objectifs exerce une influence cruciale sur leurs propres attitudes. À l'aide de la méthode de Baron et Kenny (1986) destinée à évaluer statistiquement les effets médiateurs, nous avons cherché à étudier l'action des filtres perceptifs et les représentations chez les sujets apprenants, ici les adolescents. Nous avons testé ainsi les chemins de la transmission maternelle des attitudes d'acculturation sur les adolescents pour les trois attitudes d'acculturation (intégration, assimilation, et séparation) en posant deux questions: les attitudes d'acculturation de l'adolescent sont-elles en lien avec les attitudes d'acculturation de la mère et ses objectifs d'acculturation? De quelle façon les perceptions qu'a l'adolescent des attitudes et des objectifs d'acculturation de sa mère médiatisent-elles les influences respectives de ces attitudes et de ces objectifs d'acculturation?

L'analyse corrélationnelle montre que les attitudes d'acculturation des adolescents sont toutes liées à leur perception des attitudes de leur mère et à celle de ses objectifs. Ces perceptions sont cohérentes avec les attitudes d'acculturation de la mère et à ses objectifs, sauf en ce qui concerne l'assimilation, pour laquelle il n'y a pas de corrélation entre les attitudes d'assimilation de la mère et la perception qu'en a l'adolescent. L'influence des objectifs d'acculturation de la mère (deuxième chemin du tableau 4) est claire mais indirecte pour les trois attitudes d'acculturation: elle est de fait complètement médiatisée par les perceptions qu'a l'adolescent des objectifs d'acculturation de sa mère. L'influence des attitudes d'acculturation de la mère (premier chemin du tableau 4) varie, par contre, selon les attitudes. L'influence des attitudes d'intégration de la mère est complètement médiatisée par les perceptions de l'adolescent. Les attitudes d'assimilation de la mère n'ont aucun lien, ni direct ni indirect, avec les attitudes d'assimilation de l'adolescent, tandis que les attitudes de séparation de la mère ont un lien direct et indirect (médiatisé à $49 \%$ ) avec les attitudes de l'adolescent, le poids de l'influence directe étant de 0,30. 
En somme, les objectifs d'acculturation de la mère exercent une influence claire sur les attitudes de l'adolescent. Si les adolescents perçoivent adéquatement ces objectifs, le filtrage cognitif qui s'opère joue un rôle important dans le processus de transmission. Plus que les objectifs de la mère, ce sont les perceptions de ces objectifs qui exercent une influence réelle. L'influence des attitudes d'acculturation de la mère sur les attitudes d'acculturation de l'adolescent est plus mitigée, elle varie selon les orientations. Les attitudes d'assimilation n'ont aucune influence, alors que les attitudes de séparation ont une influence est à la fois directe et indirecte. De ce fait, il semble bien que, là encore, les transmissions soient plus claires pour les aspects saillants de l'identité culturelle, à savoir ceux qui concernent la culture d'origine.

Tableau 4. Les chemins de la transmission des attitudes d'acculturation : rôle des perceptions des attitudes de l'autre

\begin{tabular}{|c|c|c|c|c|c|c|}
\hline & \multicolumn{2}{|c|}{ Intégration } & \multicolumn{2}{|c|}{ Assimilation } & \multicolumn{2}{|c|}{ Séparation } \\
\hline Pas & 1 & 2 & 1 & 2 & 1 & 2 \\
\hline \multicolumn{7}{|c|}{$1^{\text {er }}$ chemin : influence des attitudes de la mère en fonction des perceptions de l'adolescent } \\
\hline Attitude de la mère & $0,22 *$ & ns & ns & & $0,65 * * *$ & $0,30 * *$ \\
\hline $\begin{array}{l}\text { Perception des attitudes de la } \\
\text { mère }\end{array}$ & & $0,58 * * *$ & & $0,45 * * *$ & & $0,53 * * *$ \\
\hline$R 2$ & $0,05 *$ & $0,24 * * *$ & $n s$ & $0,20 * * *$ & $0,42 * * *$ & $0,59 * * *$ \\
\hline$\%$ de médiation & & $100 \%$ & & - & & $49 \%$ \\
\hline \multicolumn{7}{|c|}{$2^{\mathrm{e}}$ chemin : influence des objectifs de la mère en fonction des perceptions de l'adolescent } \\
\hline Objectifs de la mère & $0,28 *$ & ns & $0,32 * *$ & ns & $0,62 * * *$ & ns \\
\hline $\begin{array}{l}\text { Perception des objectifs de la } \\
\text { mère }\end{array}$ & & $0,70 * * *$ & & $0,60 * * *$ & & $0,90 * * *$ \\
\hline $\begin{array}{l}R 2 \\
\% \text { de médiation }\end{array}$ & 0,07 * & $\begin{array}{r}0,37 * * * \\
100 \%\end{array}$ & $0,10 * *$ & $\begin{array}{l}0,40 * * * \\
100 \%\end{array}$ & $0,40 * * *$ & $\begin{array}{l}0,74 * * * \\
100 \%\end{array}$ \\
\hline
\end{tabular}

Études sur l'influence conjuguée de l'environnement social et familial dans le processus de transmission culturelle

Dans les quatrième et cinquième recherches, nous avons spécifiquement examiné l'influence conjuguée de différents aspects de l'environnement social et familial dans le processus de transmission de l'identité nationale et culturelle et des valeurs familiales.

\section{Étude sur les rôles respectifs de l'environnement social et des parents}

Dans la quatrième recherche, nous avons pris en compte des facteurs provenant de l'environnement social et des facteurs provenant de la famille selon deux sources, les perceptions de l'enfant et les perceptions des parents. Du côté de l'environnement social, nous avons la densité ethnique à l'école, les relations avec les amis (proportion de Français parmi les amis) et la discrimination perçue. Du côté de la famille, nous avons interrogé les adolescents sur leur attachement à la culture de leurs parents, leur perception d'une entente à propos de leur culture avec chacun des deux parents et leur degré d'intimité avec chacun des parents pour partager leurs secrets et points de vue. Nous avons également interrogé chacun des deux parents à propos de leurs conversations avec l'adolescent autour de la culture ainsi que de leurs propres pratiques d'enculturation ethnique et française. Parmi les pratiques d'enculturation, nous avons distingué les pratiques d'enculturation pragmatique orientées vers les comportements adaptatifs et les savoirs concrets des pratiques d'enculturation émotionnelle orientées vers le savoir-être, les styles émotionnels et les valeurs (voir Sabatier, 2008). Les entrevues se sont déroulées au domicile des triades avec des intervieweurs de la même origine ethnique et ont concerné 365 adolescents (moyenne d'âge est de 15 ans et demi) de seconde génération vivant en France, 356 mères et 292 pères. 
Tableau 5. Influences relatives des variables de l'environnement social et de la famille sur les composantes de l'identité culturelle

\begin{tabular}{|c|c|c|c|c|}
\hline & \multicolumn{2}{|c|}{ Affirmation } & \multicolumn{2}{|c|}{ Exploration } \\
\hline & ethnique & française & ethnique & française \\
\hline \multicolumn{5}{|l|}{ Variables de l'environnement } \\
\hline$\%$ amis français & $-0,10(+)$ & 0,13 & & \\
\hline Discrimination perçue & & $-0,27 * * *$ & $0,12 \ddagger$ & \\
\hline \multicolumn{5}{|l|}{ Relation parent- adolescent } \\
\hline \multicolumn{5}{|l|}{ Réponses des adolescents } \\
\hline Attachement à la culture des parents & $0,48 * * *$ & & $0,35 * * *$ & \\
\hline Intimité - père & & $0,22 * * *$ & $0,16 * *$ & $0,24 * * *$ \\
\hline Harmonie culturelle avec père & & $-0,15 *$ & $-0,13 *$ & $-0,15 *$ \\
\hline \multicolumn{5}{|l|}{ Réponses des parents } \\
\hline \multicolumn{5}{|l|}{ Pratiques d'enculturation } \\
\hline \multicolumn{5}{|l|}{ De la mère } \\
\hline française émotionnelle & & $0,21 * *$ & & \\
\hline pragmatique ethnique & $0,18 * *$ & & & \\
\hline conversation & & & $0,20 * * *$ & \\
\hline \multicolumn{5}{|l|}{ Du père } \\
\hline pragmatique française & & $0,14 *$ & $-0,13 *$ & \\
\hline
\end{tabular}

$\ddagger p<0,10 ; * p<0,05 ; * * p<0,01 ; * * * p<0,001$

Les résultats montrent que les trois types d'influence, l'environnement, la relation parent-adolescent perçue par les adolescents et les pratiques d'enculturation exercent une influence sur la construction de l'identité culturelle tant ethnique que nationale et sur les deux composantes de l'identité, l'affirmation et l'exploration. L'environnement (relations avec les pairs et perception de la discrimination) explique environ $4 \%$ de la variance, la relation perçue avec les parents entre 6 et $22 \%$ et les pratiques d'enculturation parentale entre 5 et $17 \%$ selon l'orientation culturelle et la composante de l'identité. Parmi les types d'influence, la perception des adolescents de la relation avec leurs parents apparaît donc comme l'élément le plus influent, même si cet attachement s'inscrit au sein de la société et est influencé par les pratiques de socialisation. Une relation de qualité, particulièrement autour de la culture, est essentielle pour l'acquisition tant de l'identité ethnique que de l'identité nationale. L'attachement à la culture des parents est fondamental pour les deux composantes de l'identité ethnique (affirmation et exploration). La relation perçue avec le père est cruciale pour l'acquisition de l'identité nationale et pour l'exploration de l'identité, et l'analyse de sa dynamique est instructive. L'endossement de l'identité française et l'exploration de l'identité ethnique et nationale sont favorisées par la possibilité d'une certaine intimité avec le père, c'est-à-dire une certaine proximité relationnelle combinée à la possibilité d'exprimer des points de vue divergents avec le père sur la culture. Autrement dit, une relation franche avec le père, à la fois proche et divergente, est favorable à la transmission de l'identité nationale.

Etude sur le rôle du Zeitgeist dans la transmission des valeurs d'obligations familiales

Enfin, dans la cinquième recherche, qui a impliqué dix pays, dix-huit groupes culturels, 1252 dyades adolescentsparents immigrants et 726 dyades adolescents-parents "nationaux", nous avons examiné l'endossement des valeurs d'obligations familiales des adolescents en prenant en compte le climat social des pays et des cultures et les valeurs propres (voir Vedder, Berry, Sabatier et Sam, 2009). Nous nous sommes demandé dans quelle mesure les adolescents tirent leurs idées sur les obligations familiales du climat général de la société (le Zeitgeist) ou les fondent plutôt sur le climat spécifique qui règne au sein de leur groupe culturel. Les valeurs peuvent provenir des parents de l'adolescent (transmission parentale), du climat qui règne au sein du groupe des pairs (que ce soit ceux du groupe ethnique ou ceux de l'ensemble du pays), ainsi que du climat qui règne au sein des groupes de parents (ethniques ou de l'ensemble du pays). 
Nous avons examiné les influences respectives du climat social (Zeitgeist) qui règne au niveau plus large de la société, incluant tous les groupes qui la composent en ce qui concerne l'endossement des valeurs relatives aux obligations familiales (Zeitgeist national), et du climat social qui règne de façon plus spécifique au sein du groupe ethnique et culturel des individus et au niveau intrafamilial (Zeitgeist ethnique).

Le questionnaire sur les obligations familiales comprenait huit questions utilisant une échelle de Likert à 5 points, ces questions portant à la fois sur l'organisation hiérarchique de la famille et sur les normes de solidarité, par exemple : "L'autorité à l'intérieur de la famille doit être clairement définie et son exercice ne doit pas être remis en question » ou " C'est la responsabilité de l'enfant de faire attention à ses parents quand ils ont besoin d'aide ».

La mesure du Zeitgeist se fonde sur les travaux fondateurs de Glass et Polisar (1987), qui se sont interrogés sur les corrélations entre les générations et ont cherché à distinguer les effets de cohorte des effets de transmission intrafamiliale. Pour cela, en partant de leur base de données, ils ont associé à chaque famille des membres fictifs (un pour les enfants et un pour les parents). Ces membres fictifs provenaient de la banque de données et ont été associés au hasard, en s'assurant qu'aucun sujet n'apparaisse plus d'une fois et que l'adolescent et le parent fictifs ne proviennent pas de la même famille. Cette procédure a été suivie par d'autres chercheurs (Boehnke, 2001; Roest, Dubas, Gerris et Engels, 2009).

Tableau 6. Transmission des valeurs d'obligation familiale

\begin{tabular}{|c|c|c|c|c|}
\hline Niveau d'influence & 1 & 2 & 3 & 4 \\
\hline \multicolumn{5}{|l|}{ Immigrants } \\
\hline Obligation familiale du parent & $0,33 * * *$ & $0,30 * * *$ & $0,30 * * *$ & $0,30 * * *$ \\
\hline \multicolumn{5}{|l|}{ Zeitgeist ethnique } \\
\hline Pair fictif ethnique & & $0,14 * * *$ & $0,14 * * *$ & $0,13 * * *$ \\
\hline Parent fictif ethnique & & $0,06 *$ & $0,06 *$ & $0,06^{*}$ \\
\hline \multicolumn{5}{|l|}{ Zeitgeist national } \\
\hline Pair fictif national & & & 0,05 & 0,05 \\
\hline Parent fictif national & & & 0,02 & 0,02 \\
\hline Scolarité des parents & & & & $-0,03$ \\
\hline$\%$ temps de vie au pays d'accueil & & & & $-0,11 * * *$ \\
\hline$\Delta R^{2}$ & 0,11 & 0,03 & 0,00 & ${ }^{0,} 01$ \\
\hline \multicolumn{5}{|l|}{ " Nationaux " } \\
\hline Obligation familiale du parent & $0,33 * * *$ & $0,31 * * *$ & $0,30 * * *$ & $0,30 * * *$ \\
\hline \multicolumn{5}{|l|}{ Zeitgeist ethnique } \\
\hline Pair fictif ethnique & & 0,04 & 0,03 & 0,03 \\
\hline Parent fictif ethnique & & $0,08^{*}$ & $0,07 *$ & $0,07^{*}$ \\
\hline \multicolumn{5}{|l|}{ Zeitgeist national } \\
\hline Pair fictif national & & & 0,03 & 0,03 \\
\hline Parent fictif national & & & $0,07 *$ & $0,07^{*}$ \\
\hline Scolarité des parents & & & & $-0,02$ \\
\hline$\Delta R^{2}$ & 0,11 & 0,01 & 0,01 & 0,00 \\
\hline
\end{tabular}

$* p<0,05 ; * * p<0,01 ; * * * p<0,001$

Les résultats montrent, pour les deux groupes d'adolescents (nationaux et immigrants), une transmission claire des valeurs d'obligations familiales de la part des parents, en conjonction avec une influence modeste du Zeitgeist des parents du même groupe ethnique. Dans le cas des immigrants, les adolescents sont en outre influencés par le Zeitgeist de leurs pairs du même groupe culturel qu'eux. Ce modèle montre l'influence primordiale des parents, mais souligne aussi pour les adolescents issus de l'immigration l'importance du climat général qui règne dans leur propre culture, au niveau de leurs pairs et de leurs parents. L'influence du climat général de la société ne semble pas avoir d'importance, contrairement à ce que les représentations populaires sur l'adaptation laissent croire. C'est le climat qui règne au sein du groupe ethnique, aussi bien au niveau des adolescents que des parents, qui amplifie la transmission parentale. 


\section{Conclusion}

Cet ensemble de cinq recherches, fondées sur l'examen attentif des interrelations entre différentes sources d'influence sociale des attitudes, des valeurs et des identités, apporte un éclairage sur les processus de transmission culturelle au sein des familles immigrées. Ces processus mériteraient d'être plus largement étudiés afin de faciliter l'adaptation des familles et de leurs enfants, de mettre en valeur les efforts d'un grand nombre de parents et d'affiner les programmes de prévention.

La transmission apparaît comme un processus complexe qui implique la participation de plusieurs acteurs, le sujet apprenant et le socialisateur, ainsi que l'interaction avec la société environnante. La transmission n'est que rarement une reproduction à l'identique. Les adolescents endossent des valeurs généralement moins traditionnelles et davantage orientées vers la société d'accueil ou davantage individualistes que leurs parents. Néanmoins, il ne s'agit pas d'un fossé de génération au sens strict. On observe plutôt une transmission ouverte des valeurs qui laisse place à la création et aux initiatives.

Dans le cas des cultures minoritaires, la transmission est biculturelle : elle se fait pour les valeurs, attitudes et identités orientées vers la culture d'origine mais aussi pour celles orientées vers la société d'accueil. Les parents transmettent des valeurs et des identités de la société d'accueil. Ces deux orientations sont compatibles, et les parents sont actifs dans un processus biculturel. Cependant, les attitudes plus marquantes, celles qui sont plus faciles à saisir et à définir et celles qui distinguent les parents de la société environnante, c'est-à-dire celles de la culture d'origine ou de la culture régionale, sont plus faciles à transmettre, elles sont plus faciles à repérer et à identifier pour l'adolescent. Ainsi, les attitudes orientées vers la culture d'origine des immigrants sont plus efficacement transmises par les parents que celles strictement orientées vers la société d'accueil, ces dernières étant de fait moins facilement cernables.

La transmission parentale implique les deux parents avec une complémentarité des rôles parentaux, la mère se montrant plus efficace pour transmettre les valeurs, attitudes et identités de la culture d'origine, et le père pour celles de la société d'accueil et du repérage de la discrimination. Cette spécificité étant relativement peu prise en compte, il est bien possible que les données de la littérature qui n'incluent qu'un seul parent (la plupart du temps la mère), ou qui interrogent l'adolescent sur les perceptions qu'il a de ses parents sans autre précision, ne fournissent des informations que partielles. L'étude des effets distincts du père et de la mère constituent l'une des originalités de mes travaux.

La qualité de la relation parent-enfant, l'attachement de l'adolescent à la culture de ses parents, la capacité des pères à établir une relation de confiance qui rende possible l'expression de points de vue divergents et les perceptions mutuelles de la mère et de l'adolescent sont autant d'éléments essentiels qui interviennent dans le processus de transmission. Du côté des adolescents (les sujets apprenants), les perceptions des attitudes de leurs parents et de la qualité du lien avec eux sont des filtres efficaces qui médiatisent l'influence des attitudes des parents sur leurs propres attitudes.

Les parents n'agissent pas de façon isolée. La transmission parentale s'inscrit dans le cadre de l'environnement général, elle est plus aisée lorsqu'il y existe une conjonction avec d'autres instances de socialisation, lorsqu'il existe d'autres sources de socialisation pour les enfants que les seuls parents et la seule famille. Ainsi, les valeurs d'obligations familiales sont d'autant mieux transmises que les adolescents peuvent puiser leurs représentations et leurs valeurs dans le bassin d'idées (le Zeitgeist) de leur groupe de pairs de la même origine qu'eux et dans celui du groupe de parents de leur origine culturelle. Les idées du groupe culturel (adolescents et parents) sont des amplificateurs de la transmission parentale, qui demeure, elle, déterminante. Le groupe de la même culture que celle des parents amplifie l'acquisition de ces valeurs.

La question de l'existence de processus différenciés pour les adolescents et les adolescentes reste ouverte. Chaque fois que la taille de nos échantillons a permis son examen, les résultats n'ont cependant pas indiqué de processus distincts valables dans tous les groupes culturels. 


\section{Références bibliographiques}

Alessandri, S. et Wozniak, R. (1987). The child's awareness of parental beliefs concerning the child: a developmental study. Child Development, 58, 316-323.

Baron, R. et Kenny, D. (1986). The moderator-mediator variable distinction in social psychological research: Conceptual, strategic, and statistical considerations. Journal of Personality and Social Psychology, 51(6), 1173-1182.

Berry, J. (2006). Acculturation: A conceptual overview. Dans M. Bornstein et L. Cote (dir.), Acculturation and parentchild relationships: Measurement and development (p. 13-30). Mahwah, NJ : Lawrence Erlbaum Associates Publishers.

Berry, J. et Sabatier, C. (2010). Acculturation, discrimination, and adaptation among second generation immigrant youth in Montreal and Paris. International journal of intercultural relations, 34(3), 191-207.

Birman, D. (2006). Measurement of the 'Acculturation Gap' in immigrant families and implications for parent-child relationships. Dans M. Bornstein et L. Cote (dir.), Acculturation and parent-child relationships: Measurement and development. (p. 113-134). Mahwah, NJ, US: Lawrence Erlbaum Associates Publishers.

Boehnke, K. (2001). Parent-offspring value transmission in a societal context: Suggestions for a utopian research design--with empirical underpinnings. Journal of Cross-Cultural Psychology Special Issue: Perspectives on cultural transmission, 32(2), 241-255.

Boutry-Avezou, V. et Sabatier, C. (2006). Family acculturation dynamics: The intergenerational transmission of acculturation attitudes issue. The case of Moroccan families in France. Communication présentée au $18^{\mathrm{e}}$ congrès international de l'Association for Cross-Cultural Psychology, Spetze.

Brown, C. et Bigler, R. (2005). Children's perceptions of discrimination : a developmental model. Child Development, 76(3), 533-553.

Child, I. (1943). Italian or American: The second generation in conflict. New Haven, CT : Yale University Press.

Cosnefroy, O. et Sabatier, C. (2011). Estimation de l'importance relative des prédicteurs dans un modèle de régression multiple. Intérêt et limites des méthodes récentes. L'Année psychologique, 111(2), 253-289.

Costigan, C. et Dokis, D. (2006). Similarities and differences in acculturation among mothers, fathers, and children in immigrant Chinese families. Journal of cross-cultural psychology, 37(6), 723-741.

De Vos, G. (1980). L'identité ethnique et le statut de minorité. Dans P. Tap (dir.), Identités collectives et changements sociaux (p. 27-38). Toulouse : Privat.

Glass, J. et Polisar, D. (1987). A method and metric for assessing similarity among dyads. Journal of marriage and the family, 49(3), 663-668.

Jasinskaja-Lahti, I. et Liebkind, K. (2001). Perceived discrimination and psychological adjustment among Russianspeaking immigrant adolescents in Finland. International journal of psychology, 36(3), 174-185.

Kelley, J. et De Graaf, N. (1997). National context, parental socialization, and religious belief: Results from 15 nations. American Sociological Review, 62(4), 639-659.

Killian, C. et Hegtvedt, K. (2003). The role of parents in the maintenance of second generation Vietnamese cultural behaviors. Sociological Spectrum, 23(2), 213-245.

Knafo, A. (2003). Contexts, relationship quality, and family value socialization: The case of parents' school ideological fit in Israel. Personal Relationships, 10(3), 371-388.

Kwak, K. et Berry, J. (2001). Generational differences in acculturation among Asian families in Canada: A comparison of Vietnamese, Korean and East-Indian groups. International journal of psychology, 36(3), 152162.

Alterstice - Revue Internationale de la Recherche Interculturelle, vol. 3, $n^{\circ} 1$ 
LaFromboise, T., Coleman, H. et Gerton, J. (1993). Psychological impact of biculturalism: evidence and theory. Psychological bulletin, 114(3), 395-412.

Lau, A., McCabe, K., Yeh, M., Garland, A., Wood, P. et Hough, R. (2005). The acculturation gap-distress hypothesis among high-risk Mexican American families. Journal of family psychology, 19(3), 367-375.

Lewin, K. (1936). Social psychological differences between the United States and Germany. Character and personality, 4, 265-293.

Marcia, J. (1966). Development and validation of ego identity status. Journal of personality and social psychology, $3(5), 551-558$.

Nauck, B. (2001). Intercultural contact and intergenerational transmission in immigrant families. Journal of crosscultural psychology, 32(2), 159-173.

Okagaki, L. et Bevis, C. (1999). Transmission of religious values: Relations between parents' and daughters' beliefs. Journal of genetic psychology, 160(3), 303-318.

Phinney, J. (1990). Ethnic identity in adolescents and adults: review of research. Psychological bulletin, 108(3), 499514.

Phinney, J. et Chavira, V. (1995). Parental ethnic socialization and adolescent coping with problems related to ethnicity. Journal of research on adolescence, 5(1), 31-54.

Phinney, J. et Ong, A. (2002). Adolescent-parent disagreements and life satisfaction in families from Vietnameseand European-American backgrounds. International journal of behavioral development, 26(6), 556-561.

Phinney, J., Horenczyk, G., Liebkind, K. et Vedder, P. (2001). Ethnic identity, immigration, and well-being: An interactional perspective. Journal of social issues, 57(3), 493-510.

Roest, A., Dubas, J., Gerris, J. et Engels, R. (2009). Value similarities among fathers, mothers, and adolescents and the role of a cultural stereotype: Different measurement strategies reconsidered. Journal of research on adolescence, 19(4), 812-833.

Sabatier, C. (1991). Les relations parents-enfants dans un contexte d'immigration : ce que nous savons et ce que nous devrions savoir? Santé mentale au Québec, 16(1), 165-190.

Sabatier, C. (1999). Enfant et milieu. Dans E. Esperet et J. Rondal (dir.), Manuel de psychologie du développement (p. 155-190). Bruxelles : Mardaga.

Sabatier, C. (2008). Ethnic and national identity among second-generation immigrant adolescents in France: The role of social context and family. Journal of adolescence, 31(2), 185-205.

Sabatier, C. et Avezou-Boutry, V. (2012). Français? Marocains? Une synthèse des deux? La transmission des identités nationale et culturelle dans une communauté marocaine de la région de Libourne (Aquitaine). Dans Y. Leanza, C. Sabatier et C. Brisset (dir.), Adaptation et socialisation des minorités culturelles en régions (p.103-124). Québec: Presses de l'Université Laval.

Sabatier, C. et Berry, J. (1994). Immigration et acculturation. Dans R.Bourhis et J.-P. Leyens (dir.), Stéréotypes, discrimination et relations intergroupes (p. 261-291). Bruxelles : Mardaga.

Sabatier, C. et Berry, J. (2008). The role of family acculturation, parental style and perceived discrimination in the adaptation of second generation immigrant youth in France and Canada. European journal of developmental psychology, 5(2), 159-185.

Sabatier, C. et Berry, J. (2009) The role of family enculturation and acculturation on perceived discrimination with second generation adolescents. The distinct role of mothers and fathers. Communication présentée au symposium Family Functioning and Psychosocial Adaptation in Immigrant Children and Youth, $16^{\mathrm{e}}$ European Conference on Developmental Psychology, Vilnius, Lituanie.

Sabatier, C. et Holveck, M. (2001). La réussite scolaire des enfants issus de l'immigration: une étude exploratoire des conditions familiales. Revue internationale de recherche en éducation familiale, 4(1), 37-61. 
Sam, D. et Virta, E. (2002). Intergenerational value discrepancies in immigrant and host-national families and their impact on psychological adaptation. Journal of adolescence, 26(2), 213-231.

Schönpflug, U. (2009). Cultural transmission: Psychological, developmental, social, and methodological aspects. New York : Cambridge University Press.

Schönpflug, U. et Bilz, L. (2009). The transmission process: Mechanisms and contexts. In Cultural transmission: Psychological, developmental, social, and methodological aspects. (p. 212-239). New York, NY : Cambridge University Press.

Troadec, B. (2011). Le rôle de la transmission sociale et culturelle. Dans B. Bellaj (dir.), Psychologies et cultures (p. 25-62). Paris : L'Harmattan.

Vatz Laaroussi, M. (2001). Le familial au coeur de l'immigration. Les stratégies de citoyenneté des familles immigrantes au Québec et en France. Paris : L'Harmattan.

Vedder, P., Berry, J., Sabatier, C. et Sam, D. (2009). The intergenerational transmission of values in national and immigrant Families: the role of zeitgeist. Journal of youth and adolescence, 38, 642-653.

Youniss, J. (1994). Rearing children for society. Dans J. Smetana (dir.), Beliefs about parenting: origins and developmental implications (p. 37-50). San Francisco : Jossey-Bass. 